\title{
Recurrent Germline Mutations of CHEK2 as a New Susceptibility Gene in Patients with Pheochromocytomas and Paragangliomas
}

\author{
Yinjie Gao, ${ }^{1}$ Chao Ling, ${ }^{2}$ Xiaosen Ma, ${ }^{1}$ Huiping Wang, ${ }^{1}$ Yunying Cui, ${ }^{1}$ Min Nie, \\ and Anli Tong ${ }^{1}$ \\ ${ }^{1}$ NHC Key Laboratory of Endocrinology (Peking Union Medical College Hospital), Department of Endocrinology, \\ Peking Union Medical College Hospital, Peking Union Medical College, Chinese Academy of Medical Sciences, \\ Beijing 100730, China \\ ${ }^{2}$ Laboratory of Clinical Genetics (Peking Union Medical College Hospital), Peking Union Medical College Hospital, \\ Peking Union Medical College, Chinese Academy of Medical Sciences, Beijing 100730, China \\ Correspondence should be addressed to Anli Tong; tonganli@hotmail.com
}

Received 26 July 2021; Accepted 22 September 2021; Published 30 September 2021

Academic Editor: Dario De Biase

Copyright (c) 2021 Yinjie Gao et al. This is an open access article distributed under the Creative Commons Attribution License, which permits unrestricted use, distribution, and reproduction in any medium, provided the original work is properly cited.

\begin{abstract}
Purpose. Recently, pheochromocytomas and paragangliomas (PPGLs) have been strongly suspected as hereditary tumors, as approximately $40 \%$ of patients carry germline mutations. In the cancers where defects occur to corrupt DNA repair and facilitate tumorigenesis, a CHEK2 strong association has been observed. Therefore, the purpose of this study was to investigate the effect of CHEK2 mutations for its possible pathogenicity in PPGLs. Methods. Four patients with CHEK2 mutations were recruited, as previously detected by the whole exome sequencing. Sanger sequencing was used to verify the germline mutations as well as the loss of heterozygosities (LOHs) in their somatic DNAs. Immunohistochemistry was used to analyze the expression of CHEK2 and its downstream target p53 Ser20 (phosphorylated p53). Results. The average age of studied patients was $44.25 \pm 11.18$ years, at the time diagnosis. One patient had multiple tumors which recurred quickly, while two patients had distant metastasis. None of the patient had any relevant family history. Four germline CHEK2 mutations were identified (c.246_260del; c.715G>A; c. $1008+3 \mathrm{~A}>\mathrm{T}$; and c.1111C $>\mathrm{T}$ ). All the patients were predicted to have either pathogenic or suspected pathogenic mutations. There was no LOH of CHEK2 gene in somatic DNAs found. Additionally, neither CHEK2 proteins nor its downstream target p53 Ser20 were expressed in the tumor tissues. The inactivation of CHEK2 leads to the decrease in the p53 phosphorylation, which might promote tumorigenesis. Conclusions. For the first time, CHEK2 was identified as a susceptibility gene for PPGLs. However, the penetrance of CHEK2 gene with genotype-phenotype correlation needs to be investigated.
\end{abstract}

\section{Introduction}

The neuroendocrine tumors arising in the chromaffin cells of adrenal medulla are termed as pheochromocytomas (PCCs), whereas the extra-adrenal tumors originating in the chromaffin cells from the sympathetic and parasympathetic ganglia are known as paragangliomas (PGLs) [1]. PCCs and PGLs (PPGLs) affects around 2-5 patients/million/year, with the prevalence of about $1 / 300000$ to $1 / 100000$ for general population [2]. In the recent years, molecular pathogenesis of this group of lesions has advanced significantly. Almost $40 \%$ of PPGLs patients carry germline mutations in a growing list of genes including SDHA, SDHB, SDHC, SDHD, SDHAF2, VHL, RET, MAX, TEMEM127, FH, NF1, and KIF1B [3, 4]. Besides, genes such as EGLN1, EGLN2, MDH2, SLC25A11, MERTK, DLST, and KMT2D are also shown to be related to PPGLs [5-10]. It is noteworthy that the majority of individuals with clinical features such as family history of PPGLs, multiple tumors, and an early age of onset might be indicative of a hereditary onset, but they lack mutations in any of the known PPGLs susceptibility genes.

Cell cycle checkpoint kinase 2 (CHEK2) is located in chromosome 22q12.1, which encodes multifunctional kinase 
crucial for cell cycle regulation, DNA repair, and apoptosis [11]. In response to DNA damage, CHEK2 is required for bridging between ataxia telangiectasia mutated (ATM) kinase with its downstream checkpoint effectors; therefore, CHEK2-deficient patients may have corrupt DNA repair and conserved mutations which ultimately facilitate tumorigenesis [12]. However, as candidate tumor suppressor, CHEK2 contributes to molecular pathogenesis in various human malignancy. Thereby, heterozygous CHEK2 gene germline mutations have been observed in patients with the Li-Fraumeni cancer-predisposition syndrome (LFS), with other cancers such as breast cancer, colon cancer, thyroid cancer, bladder cancer, ovarian cancer, gastric cancer, renal cancer, and prostate cancer [13]. Hence, CHEK2 is speculated to be a low-penetrance, multiorgan cancer susceptibility gene.

Recently, whole exome sequencing (WES) technology has been employed to detect germline variations of 121 patients who did not have mutations on definite pathogenic genes. In our previous report, the use of next-generation sequencing (NGS) covering SDHA, SDHB, SDHC, SDHD, SDHAF2, VHL, RET, MAX, TEMEM127, FH, NF1, and KIF1B was analyzed in the cohort with 314 PPGL patients [3]. Among them, four patients showed CHEK2 gene heterozygous mutations. However, definitive validation of CHEK2 gene was required to ascertain it as a new candidate susceptibility gene in PPGLs and for the potential value for genetic risk assessment, prognosis, and surveillance. Therefore, this present study aims to investigate the effect of CHEK2 mutations on DNA-damage pathway and to assess its possible pathogenicity in PPGLs.

\section{Materials and Methods}

2.1. Patients. Out of PPGLs cohort, four patients (Patients 1 , 2,3 , and 4) had variants of CHEK2 gene as detected by the WES. The PPGLs cohort was recruited from the Peking Union Medical College Hospital between November 2007 and June 2013 (the detailed data of all 121 patients who received WES are not provided in this study). The collected blood samples and formalin fixed paraffin embedded (FFPE) tumor tissues and sections were collected after obtaining the written informed consent from the patients. The approval of the study was granted by the medical ethics committee of the hospital, and the results of this research were also agreed to be published. The DNAs from the peripheral blood leukocytes (Omega Blood DNA Midi Kit, Omega Bio-Tek, USA) and FFPE tumor tissues (Quick-DNATM FFPE Kit, ZYMO RESEARCH, USA) were obtained using a standard procedure from the patients having CHEK2 mutations.

2.2. Sanger Sequencing of CHEK2 Gene. The four mutations of CHEK2 gene detected by WES were verified by the PCR amplification in combination with Sanger sequencing. The PCR primers and amplification methods are shown in Table 1. For distinguishing the sequence of CHEK2 with the highly homologous pseudogenes (CHEK2P1-5) from exon 11 to exon 15, we used nested PCR amplification for detecting mutation on exon 11 of Patient 4 . All the sequences were studied for the mutations, by comparing them with the reference sequence of the CHEK2 gene (NM_007194.4 and NP_009125.1) through the NCBI website.

2.3. Loss of Heterozygosity ( $\mathrm{LOH}$ ) of CHEK2 in Tumor Tissue. PCR amplification and Sanger sequencing were done to evaluate the $\mathrm{LOH}$ of corresponding sites in somatic DNAs of patients. However, one patient FFPE sample (Patient 4) was not sufficient; therefore, only three patients FFPE tumor tissues were subjected for studying the corresponding exons of CHEK2 with mutations in somatic DNA by using the sequencing method mentioned in Table 2. The homozygous mutant for supporting that $\mathrm{LOH}$ of CHEK2 was present, and for the heterozygote means, no $\mathrm{LOH}$ in the corresponding site in somatic DNA was found.

2.4. CHEK2 Immunohistochemistry. The four patients with CHEK2 mutations were also evaluated for CHEK2 protein expression in the FFPE tumor sections by immunohistochemistry (IHC). Briefly, the sections were incubated with primary antibody of human Anti-Chk2 antibody (ab207446) (Abcam, England) at 1/100 dilution, followed by secondary incubation with the goat anti-rabbit IgG polymer (PV-9001, (ZSGB-BIO, China)) at 1/500 dilution. As a positive control, normal gland and PPGL tumor tissue with RET mutation were used.

2.5. Immunohistochemistry of Downstream Target $p 53$ Ser20. Further, CHEK2 downstream target p53 Ser20 (phosphorylated p53 by functional CHEK2) expression was evaluated by IHC. Briefly, the sections were incubated with primary antibody of human Anti-p53 Ser20 antibody (ABP50383) (Abbkine, China) at 1/200 dilution, followed by the secondary incubation with goat anti-mouse/rabbit IgG polymer (PV-8000, (ZSGB-BIO, China)) at 1/500 dilution, whereas the sections of normal gland were used as a positive control.

\section{Results}

3.1. Clinical Manifestation. The detailed clinical symptoms of the four patients with CHEK2 mutations are shown in Table 3. Among the 4 patients, three were male and 1 was female patient. The average age of the patients at the time of diagnosis was $44.25 \pm 11.18$ years old, where Patient 2 was only 30 years old at the time of PPGLs onset. Patient 1 had adrenal and paraaortic multiple tumors, which recurred in situ after surgery. Two patients had distant metastasis (Patient 2: liver metastasis and Patient 4: bone metastasis); however, no patients had family history.

3.2. Mutation Sites of CHEK2. In the studied patients, four CHEK2 germline mutations were detected, including two missenses (c.715G > A, p.E239K and c.1111C > T, p.H371Y), one deletion (c.246_260del, p.82_87del $(<50 \mathrm{bp})$ ), and one splice site mutation $(c .1008+3 \mathrm{~A}>\mathrm{T})$. The results of Sanger sequencing are shown in Figure 1. The American College of 
TABLE 1: PCR primers for four CHEK2 germline mutations.

\begin{tabular}{lrr}
\hline Primer & Upstream & Downstream \\
\hline Exon 2 & ACTTTTTAATTTTAAGTCTTG & AACGTGCCAAAAACCTGGAC \\
Exon 6 & GCCCTTGACATTTTACACT & CAAATTCATCCATCTAAGCAGG \\
Intron 9 & TTGTTTTATTGTCTTCTGTCCAA & TTTTAATCCACGGTCCCTC \\
Nested PCR & & \\
Exon $11-15$ & CGACGGCCAGTCTCAAGAAGAGGACTGTCTT & GCTATGACCATGCACAAAGCCCAGGTTCCATC \\
Exon 11 & GCAAGTTCAACATTATTCCCTTTT & ATCACCTCCTACCAGTCTGTGC \\
\hline
\end{tabular}

(a) The condition of PCR amplification for Exon 2, 6, and Intron 9 was as follows: predenaturation at $95^{\circ} \mathrm{C}$ for $5 \mathrm{~min}$, denaturation at $95^{\circ} \mathrm{C}$ for $30 \mathrm{~s}$, annealing at $54^{\circ} \mathrm{C} / 52^{\circ} \mathrm{C} / 64^{\circ} \mathrm{C}$ for $30 \mathrm{~s}$, and extension at $72^{\circ} \mathrm{C}$ for $40 \mathrm{~s}$. A total of 35 cycles were carried out, final extension at $72^{\circ} \mathrm{C}$ for 10 min. (b) The condition of nested PCR amplification for Exon 11 was as follows: (1) long-range PCR: predenaturation at $98^{\circ} \mathrm{C}$ for $5 \mathrm{~min}$, denaturation at $95^{\circ} \mathrm{C}$ for $30 \mathrm{~s}$, annealing at $68^{\circ} \mathrm{C}$ for $30 \mathrm{~s}$, and extension at $72^{\circ} \mathrm{C}$ for $3 \mathrm{~min}$. A total of 35 cycles were carried out, final extension at $72^{\circ} \mathrm{C}$ for $10 \mathrm{~min}$. Product of long-range PCR was used as a template to amplify the exon 11 using the appropriate oligonucleotide primers. (2) The condition of PCR amplification with the touch-down PCR was as follows: predenaturation at $95^{\circ} \mathrm{C}$ for $5 \mathrm{~min}$, denaturation at $95^{\circ} \mathrm{C}$ for $30 \mathrm{~s}$, annealing at $64^{\circ} \mathrm{C}$ for $1 \mathrm{~min}$ (decreased by $0.5^{\circ} \mathrm{C}$ per cycle), and extension at $72^{\circ} \mathrm{C}$ for $40 \mathrm{~s}$ in 9 cycles, and, next, predenaturation at $95^{\circ} \mathrm{C}$ for $5 \mathrm{~min}$, denaturation at $95^{\circ} \mathrm{C}$ for $30 \mathrm{~s}$, annealing at $60^{\circ} \mathrm{C}$ for $1 \mathrm{~min}$, and extension at $72^{\circ} \mathrm{C}$ for $40 \mathrm{~s}$ in $25 \mathrm{cycles}$. A total of 34 cycles were carried out, final extension at $72^{\circ} \mathrm{C}$ for $10 \mathrm{~min}$. (c) PCR products were identified by $1.5 \%$ agarose gel electrophoresis and sent to the Beijing SinoGenoMax Company for purification and sequencing. The sequencing was performed by ABI 3730XL instrument.

TABLE 2: PCR primers for CHEK2 mutations in somatic DNA from FFPE tissues.

\begin{tabular}{lcc}
\hline Primer & Upstream & Downstream \\
\hline 2 S300 & CACTGAGCTCCTTAGAGAC & CAAGATTGGCAAATCCATC \\
6 S770 & TTTGTTTTTCCCTCTAGTGGT & ATTATTTTGGGAAGTTATGAAG \\
9 S41980 & GAGCTGTTTGACAAAGTGGT & GTTTTAATCCACGGTCCCT \\
\hline
\end{tabular}

(a) The condition of PCR amplification was as follows: predenaturation at $95^{\circ} \mathrm{C}$ for $5 \mathrm{~min}$, denaturation at $95^{\circ} \mathrm{C}$ for $30 \mathrm{~s}$, annealing at $56^{\circ} \mathrm{C} / 52^{\circ} \mathrm{C} / 56^{\circ} \mathrm{C}$ for $30 \mathrm{~s}$, and extension at $72^{\circ} \mathrm{C}$ for $30 \mathrm{~s}$. A total of 35 cycles were carried out, final extension at $72^{\circ} \mathrm{C}$ for $10 \mathrm{~min}$. (b) PCR products were identified by $1.5 \%$ agarose gel electrophoresis and sent to the Beijing SinoGenoMax Company for purification and sequencing. The sequencing was performed by ABI $3730 \mathrm{XL}$ instrument.

TABLE 3: The detailed clinical manifestations of the four patients with CHEK2 mutations.

\begin{tabular}{|c|c|c|c|c|c|c|c|c|c|c|c|c|}
\hline Patient & Gender & $\begin{array}{c}\text { Age at } \\
\text { diagnose }\end{array}$ & Duration & Tumor & $\mathrm{NE}$ & $\mathrm{E}$ & DA & Past history & $\begin{array}{l}\text { Multiple } \\
\text { tumors }\end{array}$ & $\begin{array}{l}\text { Tumor } \\
\text { recurrence }\end{array}$ & $\begin{array}{c}\text { Tumor } \\
\text { metastasis }\end{array}$ & $\begin{array}{l}\text { Family } \\
\text { history }\end{array}$ \\
\hline 1 & Male & 55 & 7 & $\begin{array}{l}\text { PCC, } \\
\text { PGL }\end{array}$ & 537.43 & 3.54 & 345.60 & $\begin{array}{l}\text { Renal cyst, } \\
\text { cerebral } \\
\text { infarction }\end{array}$ & $\begin{array}{l}\text { Adrenal, } \\
\text { abdominal }\end{array}$ & Recurrence & No & No \\
\hline 2 & Male & 30 & 13 & PCC & 714.71 & 7.15 & 472.23 & No & No & No & Liver & No \\
\hline 3 & Male & 41 & 7 & PCC & 775.03 & 2.83 & 571.37 & No & No & No & No & No \\
\hline 4 & Female & 51 & 5 & PCC & 1608.66 & 4.74 & 342.87 & No & No & No & Bone & No \\
\hline
\end{tabular}

PCC: pheochromocytoma; PGL: paraganglioma; NE: 24-hour urinary norepinephrine (normal range: 16.7-40.7 $\mu \mathrm{g} / 24 \mathrm{~h}$ ); E: 24 -hour urinary epinephrine (normal range: 1.7-6.4 $\mu \mathrm{g} / 24 \mathrm{~h}$ ); DA: 24 -hour urinary dopamine (normal range: 120.9-330.6 $\mu \mathrm{g} / 24 \mathrm{~h}$ ); NE, E, and DA were the preoperative hormone levels of each patient and measured in $\mu \mathrm{g} / 24 \mathrm{~h}$. Age at diagnosis and duration of PPGL were measured in years.

Medical Genetics (ACMG) guidelines were used to predict the pathogenicity of the detected four variants. Two of the variants were evaluated as pathogenic mutations (Patient 2: c.715G > A, p.E239K; Patient 4: c.1111C > T, p.H317Y), and the other two were as suspected pathogenic mutations (Patient 1: c.246_260del, p.82_87del; Patient 3: c. $1008+3 \mathrm{~A}>\mathrm{T}$ ). The detailed information about the detected mutations and ACMG evaluations are shown in Table 4. Of note, these four patients had no other germline mutations of the confirmed susceptibility genes for PPGLs.

The evidences of pathogenicity of ACMG mentioned in this table were as follows: PS1: the same amino acid change as a previously established pathogenic variant regardless of nucleotide change; PS3: well-established in vitro or in vivo functional studies supportive of a damaging effect on the gene or gene product; PM1: located in a mutational hot spot and/or critical and well-established functional domain (e.g., active site of an enzyme) without benign variation; PM2: absent from controls (or at extremely low frequency if recessive) in Exome Sequencing Project, 1000 Genomes Project, or Exome Aggregation Consortium; PM4: protein length changes as a result of in-frame deletions/insertions in a nonrepeat region or stop-loss variants; PM6: assumed de novo, but without confirmation of paternity and maternity; PP3: multiple lines of computational evidence support a deleterious effect on the gene or gene product (conservation, evolutionary, splicing impact, etc.); PP5: reputable source recently reports variant as pathogenic, but the evidence is not available to the laboratory to perform an independent evaluation.

3.3. LOH of CHEK2 in Tumor Tissue. In the FFPE tumor tissues, the sites of three mutations detected in peripheral blood DNA were heterozygous in somatic DNAs (Note: Patient 4 had insufficient FFPE tumor tissues) (Figure 2). The results confirm that there was no LOH of CHEK2 gene in the studied patients. 


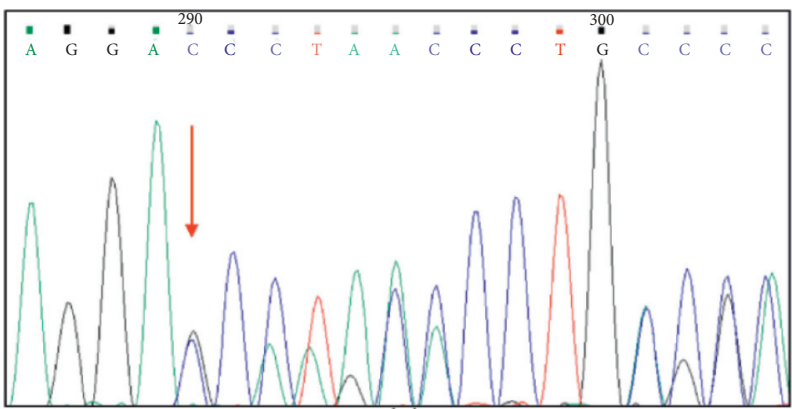

(a)

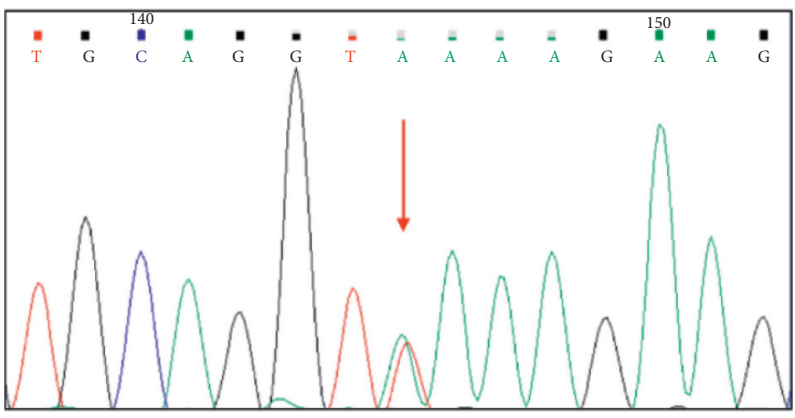

(c)

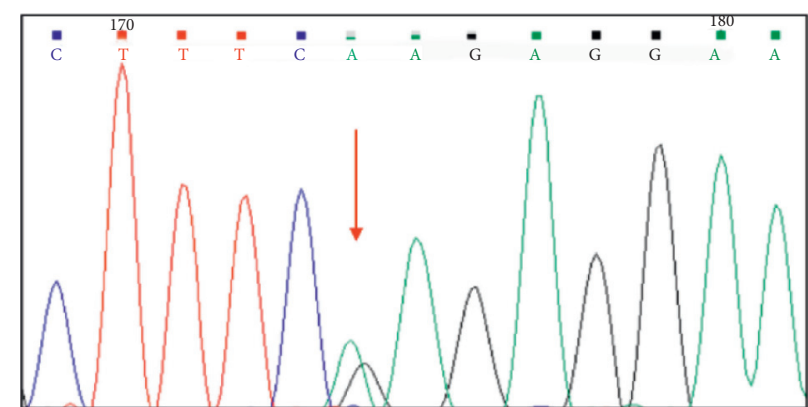

(b)

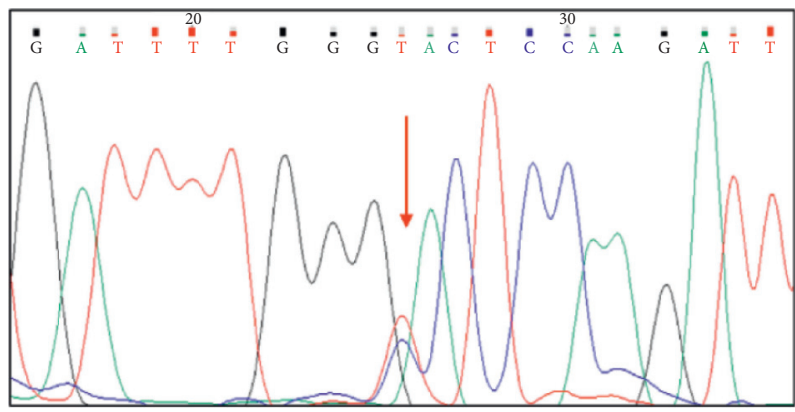

(d)

FIGURE 1: The germline CHEK2 mutations detected by Sanger sequencing of these four patients. The red arrows indicate the mutation sites, (a) for Patient 1, (b) for Patient 2, (c) for Patient 3, and (d) for Patient 4.

TABLE 4: Detailed information of mutations and ACMG evaluation.

\begin{tabular}{lccccc}
\hline Patient & Location & Base change & Amino acid change & ACMG & Pathogenicity \\
\hline 1 & Exon 2 & c. $246 \_260 \mathrm{del}$ & p. $82 \_87 \mathrm{del}$ & PM2/PM4/PM6 & Suspected pathogenic \\
2 & Exon 6 & c. G715A & p. E239K & PS1/PS3/PM1/PM6/PP3/PP5 & Pathogenic \\
3 & Intron 9 & c. $1008+3 \mathrm{~A}>\mathrm{T}$ & - & PM1/PM2/PM6 & Suspected pathogenic \\
4 & Exon 11 & c. C1111T & p. H371Y & PS3/PM1/PM6/PP3 & Pathogenic \\
\hline
\end{tabular}

3.4. Immunohistochemistry of CHEK2 Protein and the Downstream Target 553 Ser20. Compared with the normal adrenal or PPGL tumor tissue sections with RET mutation (the nucleus was positive for CHEK2 staining), the results of CHEK2 immunohistochemistry were negative in all patients except that the partial cytoplasm was weakly positive for Patient 4. This finding suggested that the CHEK2 proteins were either not expressing or inactivated in the tumor tissues (Figure 3). The results of the downstream target p53 Ser20 immunohistochemical staining were nucleus negative for these patients (except for partial cytoplasm positivity in Patients 2 and 4), as compared with positive control from normal gland tissue. These findings further confirm that the inactivation of CHEK2 could result in the decrease activity of phosphorylation of p53 protein (Figure 4). Therefore, the abnormal phosphorylation of $\mathrm{p} 53$ protein might influence the biological function and can lead to tumorigenesis.

\section{Discussion}

We previously shown that the CHEK2 gene mutations accounted for $3.3 \%(4 / 121)$ of PPGLs patients, in which pathogenic mutations of the related genes were not detected, whereas in $1.3 \%(4 / 314)$ of PPGLs patients recruited cohort from Peking Union Medical College Hospital, a frequency equivalent to a few identified PPGLs susceptibility genes including SDHA, TMEM127, MAX, and $F H$ was found [14-17]. It is noteworthy that CHEK2 gene mutations might be associated with the genetic background of PPGLS, as out of 4, three patients detected CHEK2 mutations were presented with the multiple tumors or malignant developments.

Since checkpoint defects result in the accumulation of altered genetic information and a central feature of carcinogenesis, these DNA-damage checkpoint pathways have been of interest to the field of cancer biology [18]. Among the conserved DNA-damage activated kinases identified so far, the CHEK2 plays a central role in implementing many aspects of the checkpoint response, related to the occurrence of various cancers [19]. The CHEK2 protein contains three distinct functional domains: (1) the SQ/TQ-rich, (2) the forkhead-associated, and (3) and the serine/threonine kinase domain [20]. Figure 5 shows the pattern of CHEK2 gene and the four detected mutations location. However, except for the one mutation which was present next to the SQ/TQ-rich domain, all others were in the kinase domain. 

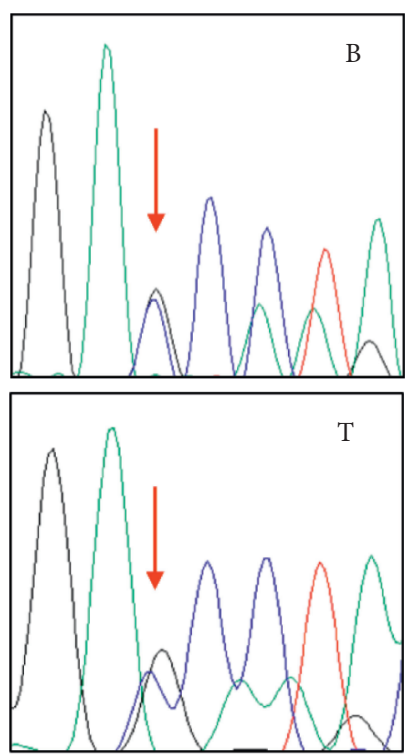

(a)
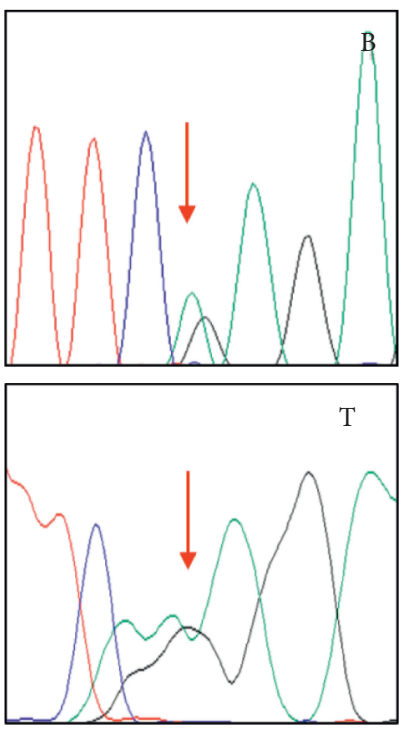

(b)
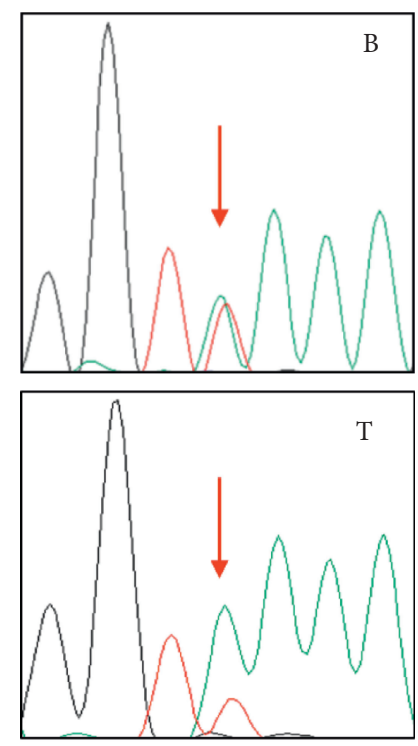

(c)

FIgURE 2: The somatic CHEK2 mutations detected of three patients compared with the germline mutation sites. The other one patient' sufficient FFPE sample was not obtained. The red arrows indicate the mutation sites, (a) for Patient 1, (b) for Patient 2, and (c) for Patient 3. " $\mathrm{B}$ " means the germline sites from peripheral blood leukocytes, and " $\mathrm{T}$ " means the somatic sites from FFPE tumor tissues.

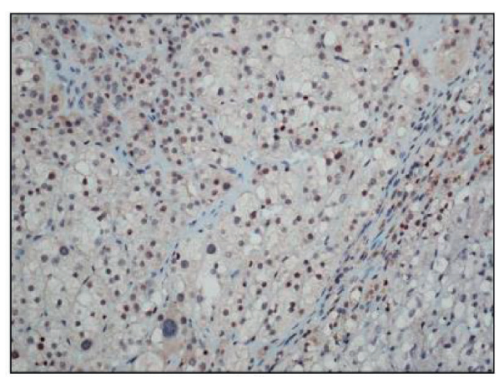

(a)

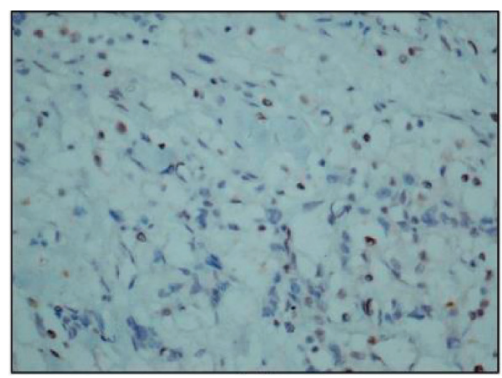

(d)

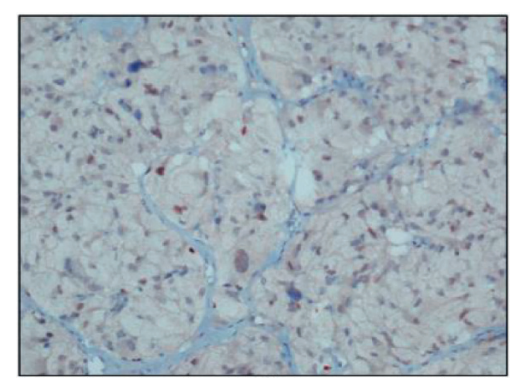

(b)

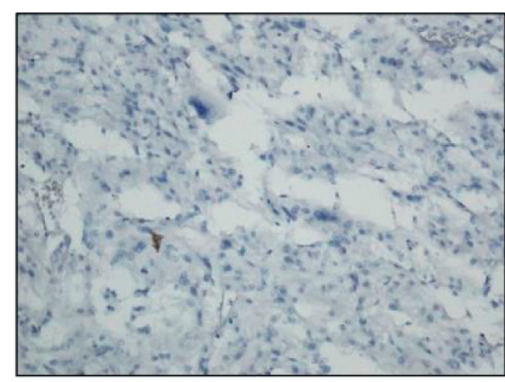

(e)

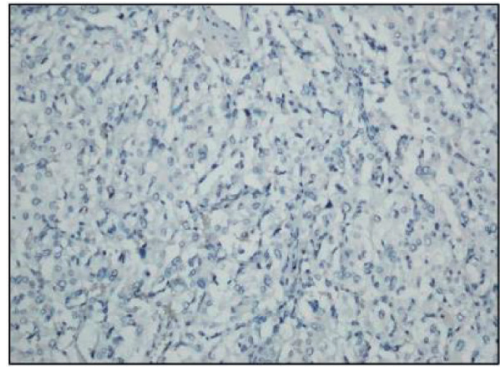

(c)

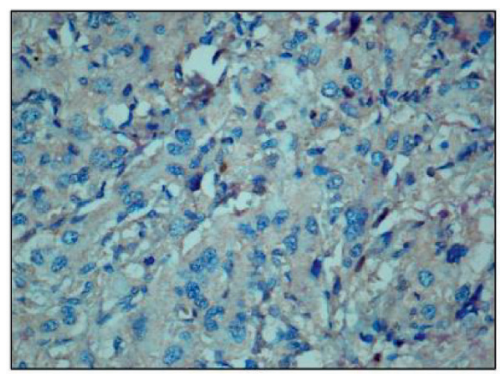

(f)

FIGURE 3: Immunohistochemical staining of CHEK2 protein. (a) Staining for normal gland (positive control: the nucleus was positive for CHEK2). (b) Staining for PPGL tumor tissue with RET mutation (positive control: the nucleus was positive for CHEK2), (c) for Patient 1 (negative for CHEK2), (d) for Patient 2 (negative for CHEK2), (e) for Patient 3 (negative for CHEK2), and (f) for Patient 4 (the nucleus was negative but partial cytoplasm was weak positive for CHEK2).

The detected four germline variants of CHEK2 in this study were causing decreased expression of the CHEK2 protein, suggesting the alterations were resulting in loss-offunction pathogenicity. Though in PPGLs, the function of CHEK2 gene has not been well characterized; however, CHEK2 role in cell proliferation and tumor suppression has been confirmed by various reports. Hong et al. established a CHEK2-1100delC mutant, which promoted the gastric cancer cell proliferation, migration, and invasion, with downregulation of E-cadherin and upregulated vimentin expression, suggesting its possible role in altered biological behavior as epithelial mesenchymal transition (EMT) [21]. 


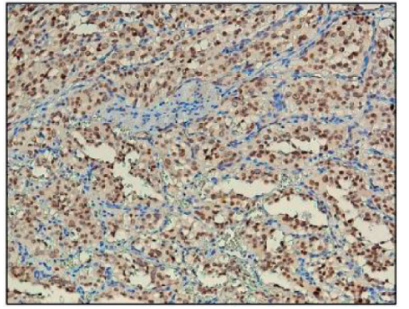

(a)

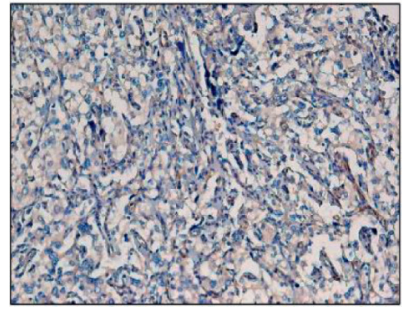

(b)

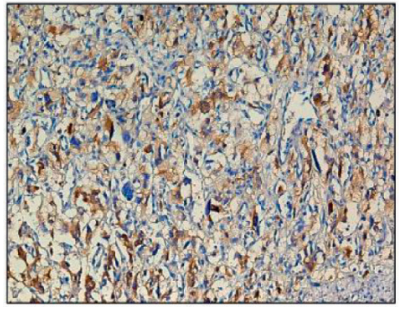

(c)

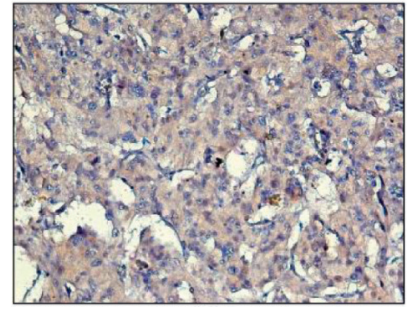

(d)

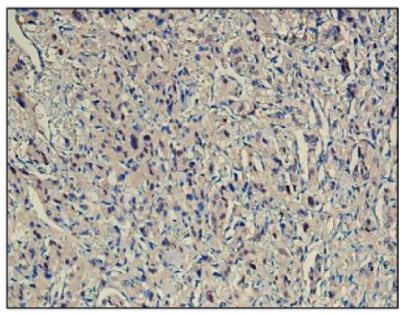

(e)

FIGURE 4: Immunohistochemical staining of the phosphorylated p53 Ser20. (a) Staining for normal gland (positive control: the nucleus was positive for p53 Ser20), (b) for Patient 1 (negative for p53 Ser20), (c) for Patient 2 (the nucleus was negative but partial cytoplasm was positive for p53 Ser20), (d) for Patient 3 (negative for p53 Ser20), and (e) for Patient 4 (the nucleus was negative but partial cytoplasm was weak positive for p53 Ser20).

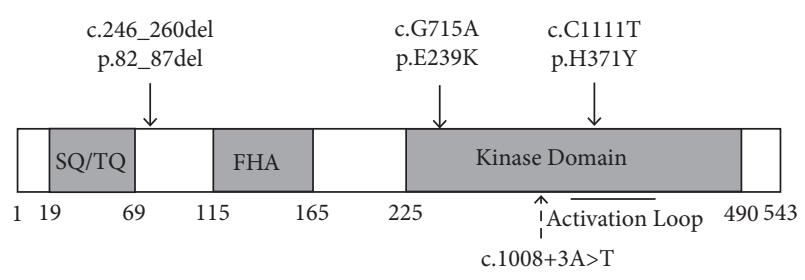

FIGURE 5: The pattern diagram of functional domain on CHEK2 gene and the location of the four detected mutations.

Another study reported the novel recurrent CHEK2-Y390 C mutant associated with increased breast cancer risk in Chinese population. The study further reported that the mutant protein's inability resulted in the lack of phosphorylation of CDC25 A Ser178 and p53 Ser20 after DNA damage, which was led to abnormal cell apoptosis and checkpoint repair [22]. In the present study, we also found that the p53 could not be phosphorylated due to CHEK2 mutations in the studied four patients, indicating the inability of variant CHEK2 proteins to efficiently bind and phosphorylate its substrates.

Among these four mutations found in the present study, two missense mutations were reported previously. In year 2003, the mutant CHEK2-E239 K was first mentioned for the prostate cancer [23]. The alteration of amino acid in the kinase activation domain significantly alter the phosphorylation of p53 in DNA-damage signaling, while the wild type CHEK2 completely retained CHEK2 kinase activity following ionizing radiation, and only $50 \%$ response was regained in the mutant group [24]. This studied mutation was later detected in patients with breast cancer and nonHodgkin's lymphoma [25, 26]. Another mutant CHEK2$\mathrm{H} 371 \mathrm{Y}$ detected in our study was confirmed as a breast cancer risk variant in 2011, for $4 \%$ of the total patients.
Approximately 50\% decrease was observed during functional analysis for the autophosphorylation, transphosphorylation, and CHEK2 activity of CHEK2-H371Y mutant [27]. The other two variants namely p.82_87del and c. $1008+3 \mathrm{~A}>\mathrm{T}$ detected in our study were not reported in databases previously. Both had high pathogenicity as evaluated by the ACMG, suggesting that these CHEK2 mutations could be deleterious as they might influence the protein structure and kinase domain. Additionally, no LOHs were detected in these corresponding sites of the studied four patients with CHEK2 mutations. Moreover, haploinsufficiency caused by dominant negative effect, or the change in protein spatial structure with the mutant amino acid folding, can lead to the abnormal function by only one allele variant $[28,29]$.

In the present study, lack of family history in four pedigrees was investigated for genes such as $M D H 2, B A P 1$, DLST, or SLC25A11 [6, 9, 10, 30]. However, among the de novo mutation or low-penetrance inheritance, the latter is frequently associated with PPGLs $[6,31]$. On the other hand, due to the advancement in genetics, germline mutations and familial syndromes are known to be associated with $8-24 \%$ of sporadic PPGLs [2]. Germline testing is now generally recommended in PPGL, and besides the potential role played in PPGLs pathogenesis, the detection of germline variants in patients clinically defined as sporadic may be helpful in finding out the existence of unknown multineoplasia hereditary diseases [32, 33].

The current study had the following limitations. First, due to the limitation of follow-up year, we did not observe the other multiple tumors in these patients with CHEK2 mutations or their family members. Second, the DNAs from blood leukocytes of patient's parents were not obtained; therefore, we could not identify if the mutations had de novo origin. Thirdly, all CHEK2 variants detected in somatic DNA 
were heterozygous; therefore, the potential mechanisms leading to the abnormal function by only one allele variant should be further researched. Lastly, in the results of IHC for CHEK2 expression, tumor or normal adrenal tissue, stromal cells, such as vascular endothelial cells, were not stained positive. These findings on CHEK2 staining were also discussed in previous studies [34-37]. Therefore only positive or negative staining of tumor cells was compared and analyzed here.

\section{Conclusions}

In conclusion, we have identified four germline variants, which functionally compromises CHEK2, suggesting CHEK2 as a susceptibility gene for PPGLs. However, due to the limited number of patients and low prevalence of the CHEK2 mutations, more cases are required for the validation of its penetrance and genotype-phenotype correlation in PPGLs.

\section{Data Availability}

Data would be available upon request from corresponding author of this manuscript.

\section{Conflicts of Interest}

The authors declare that the research was conducted in the absence of any commercial or financial relationships that could be construed as a potential conflict of interest.

\section{Acknowledgments}

This research was funded by the National Natural Science Foundation of China (Grant nos. 81770427 and 82070822) and CAMS Innovation Fund for Medical Sciences (Grant no. 2017-I2M-1-001).

\section{References}

[1] H. P. H. Neumann, W. F. Young Jr., and C. Eng, "Pheochromocytoma and paraganglioma," New England Journal of Medicine, vol. 381, no. 6, pp. 552-565, 2019.

[2] D. Sacks, D. Sacks, B. Baxter et al., "Multisociety consensus quality improvement revised consensus statement for endovascular therapy of acute ischemic stroke," International Journal of Stroke: Official Journal of the International Stroke Society, vol. 13, no. 6, pp. 612-632, 2018.

[3] X. Ma, M. Li, A. Tong et al., "Genetic and clinical profiles of pheochromocytoma and paraganglioma: a single center study," Frontiers in Endocrinology, vol. 11, Article ID 574662, 2020.

[4] J. Welander, A. Andreasson, C. C. Juhlin et al., "Rare germline mutations identified by targeted next-generation sequencing of susceptibility genes in pheochromocytoma and paraganglioma," Journal of Clinical Endocrinology \& Metabolism, vol. 99, no. 7, pp. E1352-E1360, 2014.

[5] R. A. Toledo, Y. Qin, Z.-M. Cheng et al., "Recurrent mutations of chromatin-remodeling genes and kinase receptors in pheochromocytomas and paragangliomas," Clinical Cancer Research, vol. 22, no. 9, pp. 2301-2310, 2016.
[6] L. Remacha, D. Pirman, C. E. Mahoney et al., "Recurrent germline DLST mutations in individuals with multiple pheochromocytomas and paragangliomas," The American Journal of Human Genetics, vol. 104, no. 4, pp. 651-664, 2019.

[7] C. C. Juhlin, A. Stenman, F. Haglund et al., "Whole-exome sequencing defines the mutational landscape of pheochromocytoma and identifies KMT $2 \mathrm{D}$ as a recurrently mutated gene," Genes, Chromosomes and Cancer, vol. 54, no. 9, pp. 542-554, 2015.

[8] C. Yang, Z. Zhuang, S. M. J. Fliedner et al., "Germ-line PHD1 and PHD2 mutations detected in patients with pheochromocytoma/paraganglioma-polycythemia," Journal of Molecular Medicine, vol. 93, no. 1, pp. 93-104, 2015.

[9] B. Calsina, M. Currás-Freixes, A. Buffet et al., "Role of MDH2 pathogenic variant in pheochromocytoma and paraganglioma patients," Genetics in Medicine, vol. 20, no. 12, pp. 1652-1662, 2018.

[10] A. Buffet, A. Morin, L.-J. Castro-Vega et al., "Germline mutations in the mitochondrial 2-oxoglutarate/malate carrier SLC25A11 gene confer a predisposition to metastatic paragangliomas," Cancer Research, vol. 78, no. 8, pp. 1914-1922, 2018.

[11] J. Ahn, M. Urist, and C. Prives, "The Chk2 protein kinase," DNA Repair, vol. 3, no. 8-9, pp. 1039-1047, 2004.

[12] J. Smith, L. Mun Tho, N. Xu, and D. A. Gillespie, "The ATMChk2 and ATR-Chk1 pathways in DNA damage signaling and cancer," Advances in Cancer Research, vol. 108, pp. 73-112, 2010.

[13] S. K. Aedma and A. Kasi, "Li-fraumeni syndrome," in StatPearls. edn. Treasure Island (FL): StatPearls Publishing Copyright () 2020, StatPearls Publishing LLC, Treasure Island, FL, USA, 2020.

[14] S. H. Seo, J. H. Kim, M. J. Kim et al., "Whole exome sequencing identifies novel genetic alterations in patients with pheochromocytoma/paraganglioma," Endocrinology and Metabolism, vol. 35, no. 4, pp. 909-917, 2020.

[15] S. Albattal, M. Alswailem, Y. Moria et al., "Mutational profile and genotype/phenotype correlation of non-familial pheochromocytoma and paraganglioma," Oncotarget, vol. 10, no. 57, pp. 5919-5931, 2019.

[16] H. Choi, K. J. Kim, N. Hong et al., "Genetic analysis and clinical characteristics of hereditary pheochromocytoma and paraganglioma syndrome in Korean population," Endocrinology and Metabolism, vol. 35, no. 4, pp. 858-872, 2020.

[17] G. R. Clark, M. Sciacovelli, E. Gaude et al., "Germline FH mutations presenting with pheochromocytoma," Journal of Clinical Endocrinology \& Metabolism, vol. 99, no. 10, pp. E2046-E2050, 2014.

[18] P. Apostolou and I. Papasotiriou, "Current perspectives on CHEK2 mutations in breast cancer," Breast Cancer: Targets and Therapy, vol. 9, pp. 331-335, 2017.

[19] M. K. Mustofa, Y. Tanoue, C. Tateishi, C. Vaziri, and S. Tateishi, "Roles of Chk2/CHEK2 in guarding against environmentally induced DNA damage and replication-stress," Environmental and Molecular Mutagenesis, vol. 61, no. 7, pp. 730-735, 2020.

[20] L. Zannini, D. Delia, and G. Buscemi, "CHK2 kinase in the DNA damage response and beyond," Journal of Molecular Cell Biology, vol. 6, no. 6, pp. 442-457, 2014.

[21] Y. Hong, J. Shi, Z. Ge, and H. Wu, "Associations between mutations of the cell cycle checkpoint kinase 2 gene and gastric carcinogenesis," Molecular Medicine Reports, vol. 16, no. 4, pp. 4287-4292, 2017.

[22] N. Wang, H. Ding, C. Liu et al., "A novel recurrent CHEK2 Y390C mutation identified in high-risk Chinese breast cancer 
patients impairs its activity and is associated with increased breast cancer risk," Oncogene, vol. 34, no. 40, pp. 5198-5205, 2015.

[23] X. Dong, L. Wang, K. Taniguchi et al., "Mutations in CHEK2 associated with prostate cancer risk," The American Journal of Human Genetics, vol. 72, no. 2, pp. 270-280, 2003.

[24] W. Roeb, J. Higgins, and M.-C. King, "Response to DNA damage of CHEK2 missense mutations in familial breast cancer," Human Molecular Genetics, vol. 21, no. 12, pp. 2738-2744, 2012.

[25] F. Le Calvez-Kelm, F. Lesueur, F. Lesueur et al., "Rare, evolutionarily unlikely missense substitutions in CHEK2contribute to breast cancer susceptibility: results from a breast cancer family registry case-control mutation-screening study," Breast Cancer Research, vol. 13, no. 1, p. R6, 2011.

[26] O. Havranek, P. Kleiblova, J. Hojny et al., "Association of germline CHEK2 gene variants with risk and prognosis of non-hodgkin lymphoma," PloS One, vol. 10, no. 10, Article ID e0140819, 2015.

[27] Y. Liu, J. Liao, Y. Xu et al., "A recurrent CHEK2 p.H371Y mutation is associated with breast cancer risk in Chinese women," Human Mutation, vol. 32, no. 9, pp. 1000-1003, 2011.

[28] S. B. Lee, S. H. Kim, D. W. Bell et al., "Destabilization of CHK2 by a missense mutation associated with Li-Fraumeni Syndrome," Cancer Research, vol. 61, no. 22, pp. 8062-8067, 2001.

[29] C. R. Jekimovs, X. Chen, X. Chen et al., "Low frequency of CHEK2 1100delC allele in Australian multiple-case breast cancer families: functional analysis in heterozygous individuals," British Journal of Cancer, vol. 92, no. 4, pp. 784-790, 2005.

[30] V. Maffeis, R. Cappellesso, L. Nicolè et al., "Loss of BAP1 in pheochromocytomas and paragangliomas seems unrelated to genetic mutations," Endocrine Pathology, vol. 30, no. 4, pp. 276-284, 2019.

[31] F. Schiavi, R. L. Milne, E. Anda et al., "Are we overestimating the penetrance of mutations in SDHB?" Human Mutation, vol. 31, no. 6, pp. 761-762, 2010.

[32] B. Sarkadi and A. Patócs, "Hereditary diseases predisposing to pheochromocytoma (VHL, NF-1, paraganglioma syndromes, and novel genes)," Experientia Supplementum, vol. 111, pp. 129-147, 2019.

[33] S. Nölting, N. Bechmann, D. Taieb et al., "Personalized management of pheochromocytoma and paraganglioma," Endocrine Reviews, pp. 1-41, 2021.

[34] B. Davidson, M. Bjørnerem, A. Holth, E. Hellesylt, T. E. Hetland Falkenthal, and V. A. Flørenes, "Expression, activation and clinical relevance of CHK1 and CHK2 in metastatic high-grade serous carcinoma," Gynecologic Oncology, vol. 150, no. 1, pp. 136-142, 2018.

[35] V. Staalesen, J. Falck, S. Geisler et al., "Alternative splicing and mutation status of CHEK2 in stage III breast cancer," Oncogene, vol. 23, no. 52, pp. 8535-8544, 2004.

[36] L. H. Williams, D. Choong, S. A. Johnson, and I. G. Campbell, "Genetic and epigenetic analysis of CHEK2 in sporadic breast, colon, and ovarian cancers," Clinical Cancer Research, vol. 12, no. 23, pp. 6967-6972, 2006.

[37] W. Zhao, S. Chen, X. Hou, G. Chen, and Y. Zhao, "CHK2 promotes anoikis and is associated with the progression of papillary thyroid cancer," Cellular Physiology and Biochemistry, vol. 45, no. 4, pp. 1590-1602, 2018. 